\title{
The value of histopathology of the placenta in a tertiary referral hospital in South Africa
}

\author{
Z Malusi, ${ }^{1}$ MB ChB, FCPath; P T Schubert, ${ }^{1}$ MB ChB, FCPath, MMed, MSc MedSc, MPhil, MIAC; \\ G B Theron, ${ }^{2}$ MB ChB, MMed, FCOG, BSc(Hons), MD; C A Wright, ${ }^{1,3}$ MB BCh, MMed, FCPath, FRCPath, FIAC, PhD
}

${ }^{1}$ Department of Pathology, Faculty of Medicine and Health Sciences, Stellenbosch University and National Health Laboratory Service, Tygerberg Hospital, Cape Town, South Africa

${ }^{2}$ Department of Obstetrics and Gynaecology, Faculty of Medicine and Health Sciences, Stellenbosch University, Cape Town, South Africa

${ }^{3}$ Lancet Laboratories, Johannesburg, South Africa

Corresponding author: Z Malusi (zingisamalusi@yahoo.com)

\begin{abstract}
Background. Unexplained intrauterine death (IUD) remains the most common cause of perinatal death in babies of $<1000 \mathrm{~g}$ in South Africa (SA). Information from examination of the placenta subsequent to an adverse perinatal outcome is often underutilised and placental histology can contribute to determining the cause of perinatal death and other adverse outcomes in many instances.

Objectives. To correlate placental histopathology with the clinical indication for submission and to demonstrate the value of placental histopathology in understanding adverse perinatal outcomes.

Methods. We reviewed 2 years' singleton placental histology reports at a tertiary academic hospital in the Western Cape, SA. All samples were from placentas of $>24$ weeks' gestation.

Results. The total sample $(N=822)$ comprised $60.9 \%$ live-birth placentas and $39.1 \%$ IUD placentas. In the IUD group, the cause of death was clinically unexplained in $55.9 \%$ of cases. Histopathology identified in this group included chorioamnionitis (CA) (34.5\%), maternal vascular malperfusion (32.1\%), abruptio placentae (31.5\%), delayed villous maturation (17.8\%) and toxoplasmosis, other agents, rubella, cytomegalovirus and herpes simplex (TORCH) infections (6.1\%), most commonly syphilis. No pathology was found in only $2 \%$ of IUD cases. Among live births, preterm labour accounted for $41.9 \%$ of placental submissions, of which the cause was unknown in $46.2 \%$ of cases. Clinically indicated and histologically defined CA was poorly correlated.

Conclusion. This study demonstrates the value of placental histopathology in cases of adverse perinatal outcome.
\end{abstract}

S Afr J Obstet Gynaecol 2019;25(2):64-67. https://doi.org/10.7196/SAJOG.2019.v25i2.1434

Data from the Perinatal Problem Identification Program (PPIP), in which close to $75 \%$ of all births in institutions were recorded between January 2012 and December 2013, show that unexplained intrauterine death (IUD) represents the largest category of perinatal death in babies of $<1000 \mathrm{~g}$ in South Africa (SA). ${ }^{[1]}$ In the majority of these cases, the diagnosis is based purely on clinical assessment, without autopsy or histology of the placentas. The PPIP report recommended that 'funding and research resources must be directed (at) identifying the causes of death in this group. ${ }^{[1]}$

Intrapartum asphyxia is reported as the most common cause of fresh IUDs ${ }^{[1]}$ and, along with prematurity, also as the most common cause of early neonatal death. However, intrapartum asphyxia, defined as deprivation of oxygen supply to the fetus during labour, is notoriously overdiagnosed. The only reliable predisposing factors are sentinel events such as uterine rupture, cord prolapse or abruptio placentae. ${ }^{[2]}$

Several studies, albeit performed in high-income countries, have shown that only $8-10 \%$ of cases of cerebral palsy result from intrapartum hypoxia. ${ }^{[2-4]}$ Placentas have up to $50 \%$ reserve capacity and compromised placentas may function adequately but be unable to cope with the stress of normal labour, resulting in hypoxia during delivery. ${ }^{[2,5]}$ A number of earlier studies have shown that placental lesions involving the placental vasculature (such as fetal thrombotic vasculopathy and meconium-associated vascular necrosis of the umbilical cord) or the placental parenchyma (such as diffuse chronic villitis or increased perivillous fibrin) may be associated with cerebral palsy and neurological injury at term. ${ }^{[5,6]}$ However, without histopathological examination of the placenta, such entities will not be identified and a diagnosis of intrapartum hypoxia may well be proffered.

The information gathered from placental examination after an adverse outcome of a pregnancy has been underutilised and given low priority in the past by obstetricians, neonatologists and general pathologists alike. Placental histology helps to assign and subclassify the cause of perinatal death in many cases. ${ }^{[7]}$ As there remains a reluctance to consent to perinatal autopsy, placental histology is often the only source of information regarding the cause of stillbirth or neonatal death. ${ }^{[8]}$ Such information may prove invaluable in preventing a subsequent stillbirth or identifying preventable community risk factors, which may reduce the number of unexplained cases of stillbirth. Placental histology is important in identifying maternal vascular malperfusion (MVM), infections and umbilical cord complications, ${ }^{[9,10]}$ and in cases of litigation against hospitals or obstetricians for adverse pregnancy outcomes, placental pathology offers objective insights to inform decisions.

The aim of this study was to review 2 years' placental histology records at a tertiary academic hospital in the Western Cape, SA, with a view to correlate findings with clinical diagnoses and so determine 
whether placental histopathology could assist in understanding adverse pregnancy outcome in this population.

\section{Methods}

We reviewed all singleton placentas of $\geq 24$ weeks' gestation submitted to the Division of Anatomical Pathology at Tygerberg Hospital (TBH) from deliveries at this institution between 1 January 2011 and 31 December 2012. TBH serves as a secondary and tertiary referral hospital to a population of approximately 2 million people. According to the hospital's obstetric records (unpublished), 7000 deliveries are recorded here annually.

This was a retrospective, descriptive, laboratory-based study. The placental examinations were performed in house and reported by experienced pathologists according to a standardised reporting template (available upon request). The final histological diagnosis was correlated with the clinical diagnosis where given. Approval for the study was obtained from the Health Research Ethics Committee of Stellenbosch University (ref. no. S15/10/218).

Placentas from multiple pregnancies, of $\leq 23$ weeks' gestation, or those submitted from hospitals or clinics other than TBH were excluded from the study.

Maternal data were obtained from the request forms submitted with the placenta and information available in the laboratory database. Data included maternal age, gravidity, parity, presence of so-called TORCH (toxoplasmosis, other agents, rubella, cytomegalovirus and herpes simplex) infections, HIV status and other available medical and obstetric history.

Gestational age, outcome of pregnancy (live birth, miscarriage, fetal distress or IUD) and placental data as detailed in the standardised template report and diagnosis were obtained from the laboratory reports. Patients' hospital records were not accessed.

Placental lesions were categorised according to the Amsterdam Placental Workshop Group's consensus definitions of placental lesions, ${ }^{[11]}$ as listed in Table 1.

Table 1. Definitions of placental pathology according to the Amsterdam Placental Workshop Group ${ }^{[1]}$

\begin{tabular}{|c|c|}
\hline Placental lesion & Features \\
\hline $\begin{array}{l}\text { Maternal vascular } \\
\text { malperfusion of the } \\
\text { placental bed }\end{array}$ & $\begin{array}{l}\text { Placental hypoplasia (weight below tenth } \\
\text { percentile and cord diameter }<8 \mathrm{~mm} \text { at term) } \\
\text { Infarction } \\
\text { Retroplacental haemorrhage } \\
\text { Distal villous hypoplasia } \\
\text { Accelerated villous maturation } \\
\text { Decidual arteriopathy }\end{array}$ \\
\hline $\begin{array}{l}\text { Fetal vascular } \\
\text { malperfusion }\end{array}$ & $\begin{array}{l}\text { Thrombosis } \\
\text { Segmental avascular villi } \\
\text { Villous stromal vascular karyorrhexis } \\
\text { Intramural fibrin deposition } \\
\text { Stem vessel obliteration/fibromuscular sclerosis } \\
\text { Vascular ectasia }\end{array}$ \\
\hline $\begin{array}{l}\text { Delayed villous } \\
\text { maturation }\end{array}$ & $\begin{array}{l}\text { Monotonous villous population with reduced } \\
\text { numbers of vasculosyncytial membranes for the } \\
\text { period of gestation }\end{array}$ \\
\hline $\begin{array}{l}\text { Ascending } \\
\text { intrauterine infection }\end{array}$ & Maternal or fetal inflammatory response \\
\hline $\begin{array}{l}\text { Villitis of unknown } \\
\text { aetiology }\end{array}$ & $\begin{array}{l}\text { Low- or high-grade villitis; no known aetiology; } \\
\text { usually associated with lymphohistiocytic } \\
\text { inflammation }\end{array}$ \\
\hline $\begin{array}{l}\text { Other inflammatory } \\
\text { lesions }\end{array}$ & $\begin{array}{l}\text { Eosinophilic/T-cell vasculitis, chronic } \\
\text { intervillositis or chronic deciduitis }\end{array}$ \\
\hline
\end{tabular}

\section{Data analysis}

Data were entered into a spreadsheet and exported to SPSS version 23.0 (IBM Corp., USA) for statistical analysis. Values are presented as means and standard deviations for normally distributed data, and otherwise as medians, with categorical variables expressed as percentages. Differences between variables were assessed using a chi-squared test for categorical variables or a $t$-test for quantitative variables with a normal distribution. Results were considered statistically significant if $p<0.05$.

\section{Results}

A total of 822 placentas were included in the study, with 501 (60.9\%) and 321 (39.1\%) being from live births and IUDs, respectively.

Mean maternal age was 27 years (range 14 - 45 years) and mean gestational age was 32 weeks (range 24 - 44 weeks). The mean placental weight across 814 cases (no data available for 8 cases) was $328 \mathrm{~g}$ (range 56 - $1014 \mathrm{~g}$ ).

Maternal HIV status was positive in 229 (27.9\%) cases and negative in $593(72.1 \%)$ cases.

The indications for histology requests included IUD of unknown aetiology ( $n=162 ; 19.7 \%)$, whereas preterm labour of unknown aetiology accounted for $11.8 \%$ of requests $(n=97)$. Placentas received without a clinical indication or history accounted for $5.4 \%(n=44)$ of the samples and chorioamnionitis (CA) was clinically suspected in $12.2 \%(n=100)$ of cases (Table 2$)$.

Upon comparing the clinical indication for submission with the histopathological diagnosis, abruptio placentae (AP) showed 90\% specificity and $12 \%$ sensitivity, with a positive predictive value of $50 \%$ and a negative predictive value of $76 \%$. In 42 cases, AP and CA occurred concurrently (inflammatory placental abruption).

A summary of clinical indications for IUD is given in Table 3. The most common indication was unexplained IUD $(n=162 ; 55.9 \%)$, followed by maternal hypertensive disorders ( $n=55 ; 18.9 \%$ ).

Table 4 summarises the pathology differences between samples from live births and stillbirths. Delayed villous maturation (DVM), AP, MVM, fetal vascular malperfusion (FVM) and TORCH infections were more prevalent in stillbirth placentas than in those from live births, all associated with statistically significant $p$-values.

Table 2. Clinical indication for submission of placentas $(N=822)$

\begin{tabular}{ll}
\hline Clinical indication & $\boldsymbol{n}(\%)$ \\
\hline Abruptio placentae & $27(3.3)$ \\
Chorioamnionitis & $100(12.2)$ \\
Fetal anomalies & $30(3.7)$ \\
Fetal distress & $29(3.5)$ \\
Hypoxic ischaemic encephalopathy & $28(3.4)$ \\
Maternal hypertensive disorders & $144(17.5)$ \\
Unexplained IUD & $162(19.7)$ \\
Intrauterine growth restriction & $29(3.5)$ \\
Maternal disease & $76(9.2)$ \\
Preterm labour of unknown aetiology & $97(11.8)$ \\
TORCH infection & $41(5.0)$ \\
Other & $15(1.8)$ \\
No clinical indication & $44(5.4)$ \\
IUD $=$ intrauterine death; TORCH = toxoplasmosis, other agents, rubella, cytomegalovirus and \\
herpes simplex.
\end{tabular}




\section{Discussion}

The most common indication for submission of the placenta from live births was preterm labour ( $n=210 ; 41.9 \%)$; in 97 cases (46.2\%) the cause of preterm labour was unknown. CA and maternal hypertension disorders were suspected as cause in $44(21.0 \%)$ and 18 cases $(8.6 \%)$, respectively. CA was the most common histological finding in placentas from live births $(n=154 ; 30.7 \%)$, as shown in Table 4.

Identifying the cause of preterm labour is important not only for the management of individual cases but also in context of cost implications. Predisposing and precipitating factors include CA, AP and recurrent lesions such as villitis of unknown aetiology, FVM, MVM and massive perivillous fibrin deposition (MPFD). Managing preterm neonates has considerable cost implications for both the individual and the health system and may be associated with significant morbidity.

Table 3. Clinical indications for intrauterine death $\left(N=290^{*}\right)$

\begin{tabular}{ll}
\hline Clinical indication & $\boldsymbol{n}(\%)$ \\
\hline Abruptio placentae & $4(1.4)$ \\
Chorioamnionitis & $14(4.8)$ \\
Fetal anomalies & $8(2.8)$ \\
Fetal distress & $2(0.7)$ \\
Intrauterine growth restriction & $4(1.4)$ \\
Maternal disease & $20(6.9)$ \\
Maternal hypertensive disorders & $55(18.9)$ \\
TORCH infection & $20(6.9)$ \\
Umbilical cord accident & $1(0.3)$ \\
Unexplained IUD & $162(55.9)$ \\
& \\
TORCH $=$ toxoplasmosis, other agents, rubella, cytomegalovirus and herpes simplex; \\
IUD = intrauterine death.
\end{tabular}

Fetal distress was cited as a clinical indication for placental examinations of live births. Although the clinician suggested a possible cause for this based on the clinical presentation of the mother or fetus/neonate in some cases, such as maternal hypertension $(n=144)$, the cause of the fetal distress was unknown in 29 cases (3.5\%). The most common histological findings in these cases included MVM $(n=10), \operatorname{AP}(n=5)$ and CA $(n=4)$.

In most of the IUD cases submitted for placental examinations, the cause of stillbirth was unknown ( $n=162 ; 55.9 \%)$. Insight into the reason for IUD is important to both the clinician and the parent(s), as understanding what went wrong can help to give the parent(s) closure and prevent their apportioning blame to either themselves or the attending clinician for a process that may have occurred in the antenatal period. Histopathological examination of placentas from unexplained IUDs revealed CA in $34.6 \%$ of cases $(n=56)$, followed by MVM and AP at $32.1 \%$ and $31.5 \%$, respectively. Evidence of DVM was found in $17.8 \%$ of the unexplained IUD cases and TORCH infections accounted for $6.2 \%$ of the cases $(n=10)$, the majority of which included treponemal infection. MPFD and villitis of unknown aetiology accounted for $3.1 \%$ and $4.9 \%$ of cases in this subgroup, respectively. Only five cases of unexplained IUD (3.1\%) were without pathological findings.

The correlation between clinically suspected and histologically confirmed CA in line with the poor concordance reported in literature: 100 cases were identified clinically, but 265 cases were diagnosed histologically, with a specificity of $90 \%$ and a sensitivity of $34 \%$ (the positive predictive value was $61 \%$ and the negative predictive value was $74 \%$ ). In the 154 cases of CA identified in livebirth placentas, 108 had a fetal response as manifested by funisitis/ vasculitis (70.1\%), whereas in the 111 cases of CA in IUD placentas, only 55 had a fetal response (49.5\%).

Table 4. Comparison of histopathological findings in placentas from intrauterine deaths and live births

\begin{tabular}{|c|c|c|c|c|}
\hline Histopathological finding & Outcome & Live births $(N=501), n(\%)$ & IUD $(N=321), n(\%)$ & $p$-value \\
\hline \multirow{2}{*}{ Chorioamnionitis } & Negative & $347(69.3)$ & $210(65.4)$ & 0.250 \\
\hline & Positive & $154(30.7)$ & $111(34.6)$ & \\
\hline \multirow{2}{*}{ Intervillositis* } & Negative & 489 (97.6) & $310(96.6)$ & 0.686 \\
\hline & Positive & $8(1.6)$ & $9(2.8)$ & \\
\hline \multirow{2}{*}{ Delayed villous maturation } & Negative & $460(91.8)$ & $258(80.4)$ & 0.000 \\
\hline & Positive & $41(8.2)$ & 63 (19.6) & \\
\hline \multirow{2}{*}{ Abruptio placentae/retroplacental haemorrhage } & Negative & $396(79.0)$ & $220(68.5)$ & 0.001 \\
\hline & Positive & $105(21.0)$ & $101(31.5)$ & \\
\hline \multirow{2}{*}{ Maternal vascular malperfusion } & Negative & $363(72.5)$ & $199(62.0)$ & 0.002 \\
\hline & Positive & $138(27.5)$ & $122(38.0)$ & \\
\hline \multirow{2}{*}{ Fetal vascular malperfusion } & Negative & $491(98.0)$ & $303(94.4)$ & 0.005 \\
\hline & Positive & $10(2.0)$ & $18(5.6)$ & \\
\hline \multirow{2}{*}{ TORCH infection } & Negative & $488(97.4)$ & $297(92.5)$ & 0.001 \\
\hline & Positive & $13(2.6)$ & $24(7.5)$ & \\
\hline \multirow{2}{*}{ Massive perivillous fibrin deposition } & Negative & $495(98.8)$ & $316(98.4)$ & 0.661 \\
\hline & Positive & $6(1.2)$ & $5(1.6)$ & \\
\hline \multirow{2}{*}{ Villitis of unknown aetiology } & Negative & $473(94.4)$ & $306(95.3)$ & 0.565 \\
\hline & Positive & $28(5.6)$ & $15(4.7)$ & \\
\hline \multirow{2}{*}{ Umbilical cord accident } & Negative & $498(99.4)$ & $317(98.8)$ & 0.324 \\
\hline & Positive & $3(0.6)$ & $4(1.2)$ & \\
\hline \multirow{2}{*}{ No abnormalities } & Negative & $471(94.0)$ & $314(97.8)$ & 0.010 \\
\hline & Positive & $30(6.0)$ & $7(2.2)$ & \\
\hline
\end{tabular}


DVM (also known as distal villous immaturity) is not a diagnosis but rather a reaction pattern. This condition is frequently associated with maternal metabolic conditions such as glucose intolerance, obesity and atherosclerosis. ${ }^{[12]}$ Histological evidence of DVM in a placental sample is a significant finding, because it points to an increased risk for adverse outcomes such as stillbirth, unexplained fetal growth restriction, fetal macrosomia and neurodisability. ${ }^{[11,12]}$ DVM was present in $8.2 \%$ of live births and $19.6 \%$ of IUD cases in our study.

AP is a clinicopathological diagnosis, but may be clinically silent. Histopathological diagnosis is based on macroscopic and histological findings, with the latter including a constellation of microscopic features, such as retroplacental haemorrhage, dissecting of blood into the decidua, intravillous haemorrhage and intervillous congestion or infarction. ${ }^{[10,11]}$

MVM has a multifactorial aetiology, but is frequently associated with hypertensive disorders of pregnancy, intrauterine growth restriction and recreational drug use (including tobacco smoking). Hypertensive disorders were among the most common clinical indications in our study. They are associated with retroplacental haemorrhage. ${ }^{[11,13]}$

Treponema pallidum infection (part of the TORCH infection suite) appeared to be a significant cause of morbidity and mortality in our study population, similar to results of other studies in comparable settings. ${ }^{[7,14]}$

Villitis of unknown aetiology is an uncommon but important histological finding, as it suggests a risk of recurrence in future pregnancies, with increased severity. Its diagnosis can be confirmed only by histopathology, although it may be suspected clinically owing to its association with intrauterine growth restriction, preterm labour and IUD. It is thought to be a maternal immune response to fetal antigens. ${ }^{[7,11,13]}$

There was a significant association between FVM and IUD $(p=0.005)$. FVM is associated with maternal thrombophilia, antiphospholipid antibody syndrome, abnormal cord insertion or cord obstruction and oligohydramnios. ${ }^{[13]}$

MPFD, although rare, is a notable cause of poor perinatal outcome. It is usually undiagnosed clinically and identified histologically, although the placentas may be macroscopically abnormal. It may be associated with maternal thrombophilia and antiphospholipid antibody syndrome and carries a significant recurrence risk. ${ }^{[13]}$

Histopathological examination revealed no abnormalities in only 30 of the live-birth placentas (6\%) and seven of the IUD cases $(2.2 \%)$. This demonstrates the inestimable value of placental histopathology in the case of adverse perinatal outcomes, not only for the clinician, the patient and their family, but also in the context of health economics.

\section{Study limitations}

Clinical data deemed important for the analysis of the submitted placentas were limited or absent in a number of cases. It is possible that some clinical data may have erroneously been deemed unimportant by the submitting healthcare worker.

\section{Conclusion}

Histopathological examination of the placenta provides valuable information and can reduce the number of cases of unknown aetiology in adverse perinatal outcome. If a placenta is submitted for histopathological examination, the accompanying clinical data on the request form should be as comprehensive as possible and should include, at a minimum, age, obstetric history, comorbidities, pregnancy outcome and clinical diagnosis.

Acknowledgements. We thank the Biostatistics Unit at Stellenbosch University for assistance in data analysis.

Author contributions. ZM prepared the research protocol and was responsible for data collection, arranging data analysis and preparing the manuscript. CW, GT and PS were responsible for conceptualising the study and assisted in protocol development and manuscript preparation.

Funding. None.

Conflicts of interest. None.

1. Pattinson RC, Rhoda NP. Saving Babies 2012-2013: Ninth Report on Perinatal Care in South Africa. Pretoria: Tshepesa Press, 2014

2. Ellenberg JH, Nelson KB. The association of cerebral palsy with birth asphyxia: A definition alquagmire. Dev Med Child Neurol 2013;55(3):210-216. https://doi.org/10.1111/dmcn.12016

3. Kraus FT. Perinatal pathology, the placenta, and litigation. Hum Pathol 2003;34(6):517-521. https:// doi.org/10.1016/s0046-8177(03)00227-2

4. Redline RW. Placental pathology and cerebral palsy. Clin Perinatol 2006;33(2):503-516. https://doi. org/10.1016/j.clp.2006.03.007

5. Redline RW. Cerebral palsy in term infants: A clinicopathologic analysis of 158 medicolegal case reviews. Pediatr Dev Pathol 2008;11(6):456-464. https://doi.org/10.2350/08-05-0468.1

6. Redline RW, O'Riordan MA. Placental lesions associated with cerebral palsy and neurologic impairment following term birth. Arch Pathol Lab Med 2000;124(12):1785-1791.

7. Talip Q, Theron G, Steyn W, Hall D. Total perinatally related losses at Tygerberg Hospital - A comparison between 1986, 1993 and 2006. S Afr Med J 2010;100(4):250-253. https://doi. org/10.7196/samj.3812

8. Odendaal HJ, Elliott A, Kinney HC, et al. Consent for autopsy research for unexpected death in early life. Obstet Gynecol 2011;117(1):167-171. https://doi.org/10.1097/AOG.0b013e318200cb17

9. Roberts DJ, Oliva E. Clinical significance of placental examination in perinatal medicine. J Matern Fetal Neonatal Med 2006;19(5):255-264. https://doi.org/10.1080/14767050600676349

10. Elsasser DA, Ananth CV, Prasad V, Vintzileos AM, New Jersey-Placental Abruption Study Investigators. Diagnosis of placental abruption: Relationship between clinical and histopathological findings. Eur J Obstet Gynecol Reprod Biol 2010;148(2):125-130. https://doi.org/10.1016/j. ejogrb.2009.10.005

11. Khong TY, Mooney EE, Ariel I, et al. Sampling and definitions of placental lesions: Amsterdam Placental Workshop Group consensus statement. Arch Pathol Lab Med 2016;140(7):698-713. https://doi.org/10.5858/arpa.2015-0225-CC

12. Redline RW. Distal villous immaturity. Diagn Histopathol (Oxf) 2012;18(5):189-194. https://doi. org/10.1016/j.mpdhp.2012.02.002

13. Baergen RN. The placenta as witness. Clin Perinatol 2007;34(3):393-407. https://doi.org/10.1016/j. clp.2007.03.013

14. Roberts DJ. Perinatal pathology: Practice suggestions for limited-resource settings. Arch Pathol Lab Med 2013;137(6):775-781. https://doi.org/10.5858/arpa.2011-0560-SA

Accepted 18 August 2019 